\title{
Nasopharyngeal carcinoma in situ in nasopharyngeal carcinoma
}

\author{
C W Chan, J M Nicholls, J S T Sham, P Dickens, D Choy
}

\begin{abstract}
Aims: To assess the presence of carcinoma in situ (CIS) in patients with nasopharyngeal carcinoma (NPC) and to see if the number of biopsy sites facilitates detection of CIS.

Methods: Formalin fixed, paraffin wax embedded biopsy specimens $(n=285)$ from 187 patients with NPC in 1987 were studied for the presence of CIS as well as for the histological assessment of the subtype of CIS.

Results: Fifteen $(8 \cdot 0 \%)$ patients had CIS, representing $8 \cdot 3 \%$ of all new patients with NPC and $11.6 \%$ of patients with persistent disease or relapse. CIS was undifferentiated or poorly differentiated, no cases of well differentiated squamous cell CIS were identified. There was no significant difference in the incidence of CIS when multiple endoscopic biopsy specimens were taken rather than single forceps biopsy specimens.

Conclusions: CIS can only be identified in a few patients with NPC largely because of late presentation with advanced disease at the time of diagnosis and the focal nature of the dysplastic process. The presence of dysplasia in relapses of NPC suggests that these tumours may be second growths rather than regrowths of a primary tumour.
\end{abstract}

(F Clin Pathol 1992;45:898-901)

In 1837 Durand-Fardel reported a case of nasopharyngeal carcinoma (NPC). One of the first histological classifications was detailed in 1921 by Schmincke and by Cappell in 1934 and 1938. The high prevalence of NPC in Hong Kong Chinese, was reported by Digby $e t$ $a l$ in 1940-1941. ${ }^{1}$ The World Health Organisation classification introduced in 1978 has been widely accepted and used, ${ }^{2}$ providing a useful framework for classifying NPC and comparing the results. However, precursor lesions for NPC indicative of carcinoma in situ (CIS) have not been studied in depth and there have been only a few reports looking at the pathology of these lesions. This study was therefore undertaken to look at the prevalence of nasopharyngeal carcinoma in situ occurring in cases of NPC seen within one year at our hospital. As this was a period when both multiple endoscopic and single forceps biopsy specimens were used for the diagnosis of NPC, ${ }^{3}$ the efficacy of detecting nasophar- yngeal carcinoma in situ using the two biopsy techniques was also assessed.

\section{Methods}

The slides of all biopsy cases diagnosed in 1987 as undifferentiated carcinoma, anaplastic carcinoma, and squamous cell carcinoma from the nasopharynx were retrieved and examined. If slides had faded they were restained and extra sections were cut if necessary. The specimens had all been fixed in $10 \%$ formalin, embedded in paraffin wax, and the slides stained with haematoxylin and eosin. The presence or absence of tumour was confirmed. Material from this period had been used in a previous study. ${ }^{4}$ If more than one biopsy specimen was taken from the nasopharynx during an examination it was classified as a multiple biopsy specimen. For the purpose of this study the presence of carcinoma in situ was characterised by the presence of cytological and architectural atypia affecting the whole thickness of the epithelium. Also assessed was whether such change was contiguous with the invasive tumour. The assessment was initially performed by one pathologist $(\mathrm{JN})$. To check for interobserver error the cases diagnosed as CIS were randomly distributed among those not showing CIS and shown to two other pathologists (CWC, PD). A Gomori reticulin stain was performed to confirm the confinement of atypia to the epithelium and to exclude invasion whenever necessary.

\section{Results}

From January to December 1987 a total of 285 biopsy specimens from 187 patients were positive for malignancy. Of these 187 patients, $144(77 \%)$ had a total of 228 biopsy specimens from primary growths for the initial assessment and staging of the tumour; $43(23 \%)$ patients had a total of 57 specimens to assess tumour relapse or residual disease, 12 weeks after the completion of external radiation treatment. Of the 228 specimens for initial tumour assessment, $181(79.3 \%)$ were multiple and 47 $(20.6 \%)$ were single. For the 57 biopsy specimens assessed for relapse or residual tumour, $38(67 \%)$ were multiple and $19(33 \%)$ were single.

On initial examination a total of 21 specimens from 18 patients had nasopharyngeal carcinoma in situ of which 15 were taken from primary growths and six were taken for the evaluation of relapse or persistent tumour. 


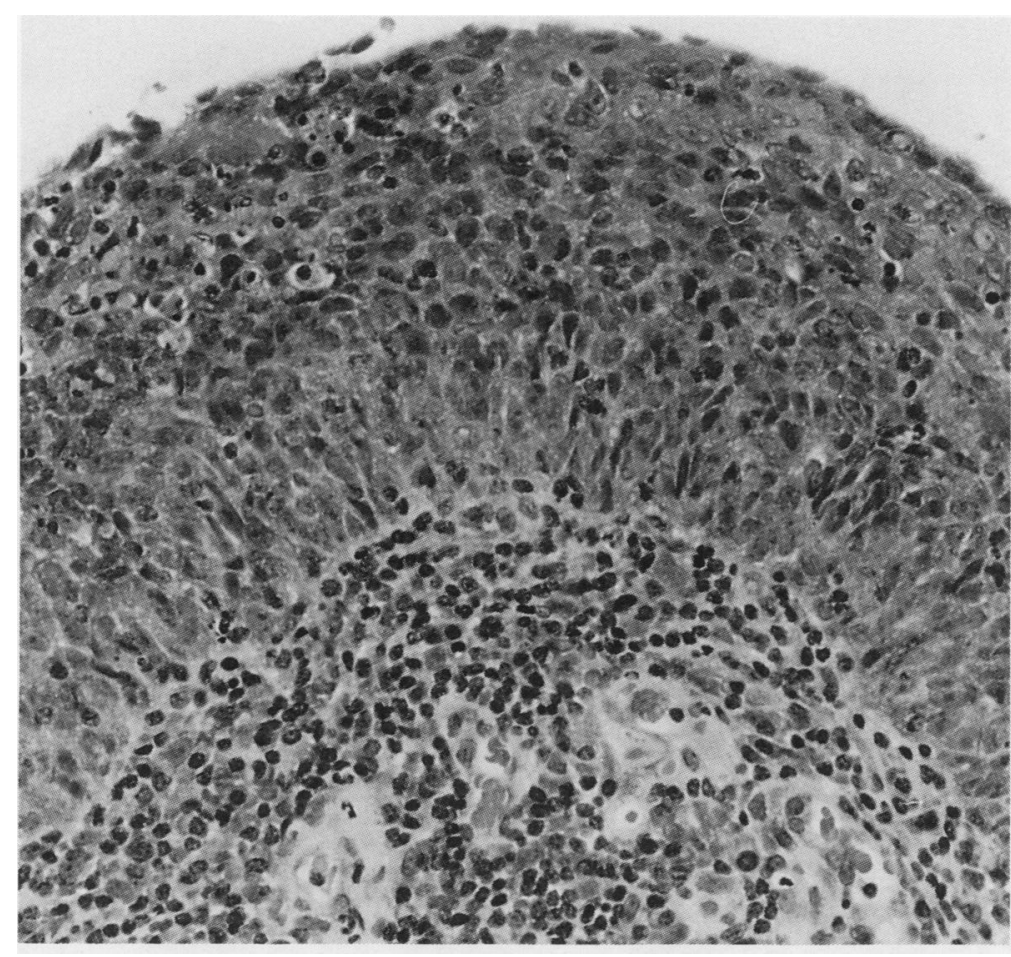

Figure 1 Carcinoma in situ of a poorly differentiated type resembling transitional cell carcinoma (haematoxylin and eosin).

Following a review by the two other pathologists (PD, CWC) the distinction between in situ change and tumour replacement of the epithelium was considered too difficult to make in two cases from primary growths and in one from a recurrent tumour. These were excluded so that in the final analysis there were 15 patients with CIS-11 with primary growths and four with relapse. One patient had nasopharyngeal carcinoma in situ in the pre-

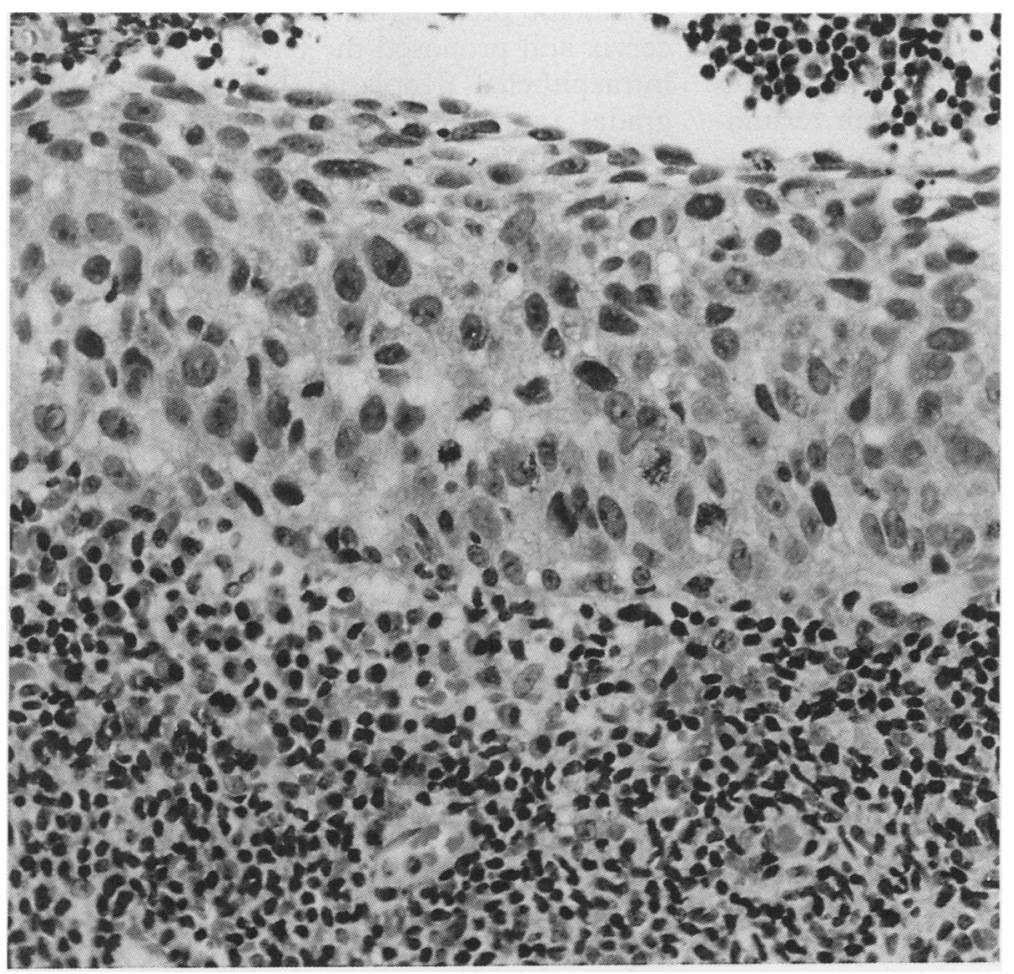

Figure 2 Carcinoma in situ of an undifferentiated type (haematoxylin and eosin). treatment biopsy specimens and in the recurrent tumour. CIS was therefore present in $8.0 \%$ of the patients with NPC. Eleven of 144 $(7 \cdot 6 \%)$ of all new patients with NPC had nasopharyngeal carcinoma in situ and five of $43(11.6 \%)$ with relapse or persistent tumour showed the presence of nasopharyngeal carcinoma in situ. Of all the 219 multiple biopsy specimens, CIS was present in $14(6.4 \%)$ and in four $(6 \cdot 1 \%)$ of the 66 single biopsy specimens. Two patients with both single and multiple biopsy specimens taken had CIS. There were no cases of well differentiated squamous cell CIS. Seven of our cases were of transitional or poorly differentiated type CIS (fig 1) and eight were analogous with an undifferentiated CIS using WHO criteria (fig 2), with an attenuated form of CIS identified (fig 3). Continuity of the CIS with tumour could only be confirmed in six cases.

\section{Discussion}

There are many types of neoplasm which may arise in the nasopharynx and these reflect the variety of normal tissue elements in this region. The most common form of cancer in this area is NPC. ${ }^{5}$ The epithelial nature of this tumour has been well characterised by electron microscopy $^{6}$ as well as by immunohistochemistry. ${ }^{7}$ There is little doubt that NPC originates in the epithelium of the nasopharynx even though continuity of tumour with the epithelium is not always seen. ${ }^{8}$ Crypt epithelial cells may have a role in NPC as they have similar histological features to undifferentiated carcinoma. Though we detected no single criterion to differentiate dysplastic from normal epithelium, we found that in the latter condition the nuclear size and irregularity, prominence of nucleoli, decrease of lymphocyte numbers and absence of differentiation in the adjacent epithelium allowed attenuated dysplasia to be diagnosed in two cases.

There has been considerable disagreement and discussion on the histopathology of NPC, but the amount of information on precursor lesions is, by comparison, minimal. One of the first reports on nasopharyngeal carcinoma in situ was by Teoh in $1957^{1}$ in which he analysed 31 necropsies between 1949 and 1955, with malignant growths in the nasopharynx. The material is currently being reanalysed using immunohistochemical techniques. In one of his patients (case 6) he noted that as well as a primary growth in the left tubal elevation sections; ". . . other parts of the nasopharynx reveal carcinoma in situ in the epithelium lining a crypt in the posterior wall ..."Unfortunately there were no photographs to document this lesion. In 1962 Liang et al looked at 500 fresh cases of NPC in the Chung Shan Medical College (in the province of Guangdong in southern China). Of their 500 biopsy cases $16(3 \cdot 2 \%)$ showed precancerous change, $17(3 \cdot 4 \%)$ showed CIS, and $34(7 \cdot 2 \%)$ showed early invasive carcinoma. No mention was made, however, of these changes in necropsy material from cases without NPC. ${ }^{9}$

Wieland referred to nasopharyngeal carcin- 
Figure 3 Carcinoma in situ of an undifferentiated type occurring in attenuated epithelium

(haematoxylin and eosin).

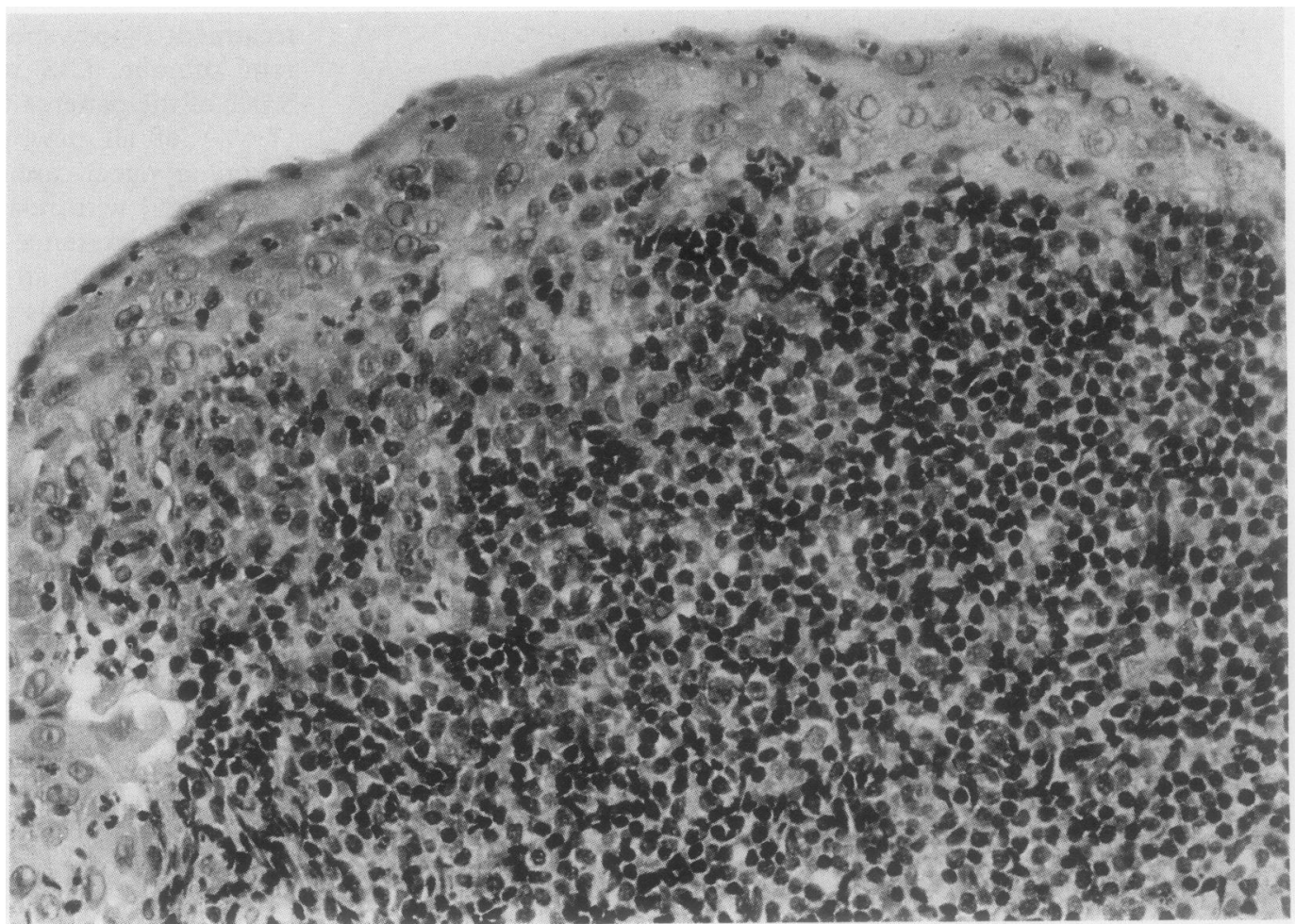

oma in situ in American referral cases of $\mathrm{NPC}^{8}$ in which he stated that in keratinising squamous cell carcinoma the tumour always occurred on the surface of the epithelium from which it originated. An apparently in situ component was frequently observed. By comparison, it was unusual to see an apparently in situ component in non-keratinising tumours; moreover, when this was seen it was difficult to prove that it represented true in situ carcinoma and not epithelial replacement from an underlying tumour.

For several years Chinese publications have referred to the precursor lesions of NPC. ${ }^{1011}$ However, they use different terminologies from those used in the West and their terminologies have mainly been based on the morphology seen in animal experiments. There are three proposed gradings of abnormal findings in the nasopharynx-namely, simple hyperplasia or metaplasia, atypical hyperplasia or metaplasia, and carcinoma in situ. This classification is therefore similar to the preneoplastic lesions seen in the bladder. Most Western researchers would consider carcinoma in situ as atypia involving the whole thickness of the epithelium when applied to squamous epithelium, but $\mathrm{Li}$ et al. ${ }^{10}$ state that CIS of the nasopharynx does not always involve the whole thickness of the epithelium and may be manifest as a few atypical, morphologically abnormal cancer cells among hyperplastic or metaplastic tissue. The results of another Chinese monograph ${ }^{11}$ found 98 cases $(8.6 \%)$ of CIS/microinvasive carcinoma among 1136 cases of NPC biopsy specimens.

The first article devoted entirely to nasopharyngeal carcinoma in situ was only published in $1986 .{ }^{12}$ The authors found an incidence of $2 \cdot 3 \%$ in 2742 biopsy specimens, and of these, the abnormal cells occupied the whole thickness of the epithelium in $85.4 \%$ of cases while in $14.9 \%$ of cases the abnormal cells occupied only part of it. Most of the lesions were found in the surface epithelium while only $18.4 \%$ were seen in the crypt epithelium. In the cases of nasophryngeal carcinoma in situ there was significantly more nuclear DNA than in normal and metaplastic squamous epithelium.

In an initial report ${ }^{13}$ and later in a monograph ${ }^{14}$ Lee et al compared the nasopharyngeal in situ and premalignant lesions to those in the cervix and proposed the term nasopharyngeal intraepithelial neoplasia (NPIN), with three grades ranging from NPIN I to NPIN III. Their data showed that $73.3 \%$ of cases of NPC were NPIN and $12.3 \%$ of surgical biopsy specimens from the normal nasopharynx were NPIN. They also found NPIN in $1.5 \%$ of a series of necropsy cases.

Our results of $8.0 \%$ are closer to the findings of Zong ${ }^{12}$ and Liang ${ }^{9}$ than those of Lee. ${ }^{14}$ The discrepancy could be that we only looked at CIS, which involves the whole thickness of the epithelium, while Lee looked at all cases with epithelial atypia. Clearly, pathologists have been unable to agree on terminology, and what is called dysplasia by some may be called atypia by others. ${ }^{15}$ We therefore chose to concentrate on looking at the high grade dysplastic lesions and found that there was reliable interobserver concordance for the diagnosis of such lesions. The reason for looking at nasophyaryngeal carcinoma in situ and classifying it is more than an academic exercise: a recent study on CIS in a selected population showed that the categorisation of precursor lesions remains an important aspect in the early detection of NPC. ${ }^{16}$ Though Lee mentioned the presence 
of koilocytic changes in biopsy material, ${ }^{14}$ other authors have not mentioned this, and we found no convincing cases in which we could identify this abnormality in our material where NPC was present.

Our study is the first to use the multiple biopsy technique to look at the presence or absence of tumour and CIS. As Zong et al mentioned in their earlier paper, by the nature of its anatomical location the nasopharynx is not easily accessible and the same degree of accuracy cannot be applied to assessing nasopharyngeal carcinoma in situ as in the cervix. ${ }^{12}$ Weiland also stated that the small size of biopsy material did not facilitate an accurate, consistent, and meaningful classification of NPC. Though no mention was made of the site of the biopsy in any of the preceding reports, we assume that they were taken straight from the tumour site and not adjacent to it. For the year studied, about $80 \%$ of the biopsy specimens for the initial assessment of NPC were multiple; for relapses the single biopsy specimens detected $33 \%$. This is because the pretreatment multiple biopsy technique was considered at that time to be important in the staging of the tumour ${ }^{17}$ and the posttreatment biopsy specimen was just to document the presence or absence of tumour. We found CIS in $6.8 \%$ of the multiple biopsy specimens and in $6.1 \%$ of the single specimens; this difference was not significant. The multiple biopsy technique may be advantageous for staging purposes but it does not promise better detection of CIS.

The prevalence of nasopharyngeal carcinoma in situ associated with invasive NPC is much less than CIN which has been used by some as a model for the nasopharynx. We attribute this to two reasons: first, we were unable to visualise the nasopharynx in as great a detail as the cervix. Secondly, and more importantly, NPC tends to present at a later stage than carcinoma of the cervix and most of the biopsy specimens of tumour do not show sufficient epithelium because this has been replaced by tumour. It should also be appreciated that CIS is an evolving lesion and the biopsy specimen represents one stage in the developing lesion. The tumour is also different from the cervical lesion in that it does not appear to go through all the stages of CIS before frank invasion develops. In this respect it is perhaps better to think of the nasopharyngeal mucosa as being more similar in behaviour to bladder rather than cervical mucosa.

One very interesting point of our study was that five cases of nasopharyngeal carcinoma in situ were found among the cases of relapse or disease persisting 12 weeks after radiotherapy had been completed. Four of these cases were in relapse while one was persistent disease. If the case of persistent disease is removed, there were four cases occurring in 24 patients with relapse $(16.7 \%)$, a figure higher than that of the primary cases. Traditionally, relapse has been considered regrowth of tumour that has not been eradicated, but our findings suggest that it could represent a second growth. Further study is being conducted in this area.

1 Teoh TB. Epidermoid carcinoma of the nasopharynx among Chinese: A study of 31 necropsies. F Pathol Bacteriol 1957;58:451-65.

2 Sham JST, Wei WI, Kwan WH, Chan CW, Choi PHK, Choy D. Fibreoptic endoscopic examination and biopsy in determining the extent of nasopharyngeal carcinoma. Cancer 1989;64:1838-42.

3 Shanmugaratnam K, Sobin LH. Histological types of upper respiratory tract tumours. In: International histological classification of tumours. No 19. Geneva: WHO 1978.

4 Nicholls JM, Sham JST, Chan CW, Choy D. Radiation therapy for nasopharyngeal carcinoma. Histological therapy for nasopharyngeal carcinoma. Histological Pathol 1992;23:742-7.

5 Shanmugaratnam $\mathrm{K}$. Histological types of nasopharyngeal carcinoma. In: de-The G, Ho Y, eds. Proceedings of international symposium on nasopharyngeal carcinoma: Etiology and Control. Lyon: International Agency for Research on Cancer, 1978:3-12.

6 Prasad U. Cells of origin of nasopharyngeal carcinoma: an electron microscopic study. I Laryngol Otol 1974; 88:1087-94.

7 Oppedal BR, Bohler PJ, Marton PF, Brandtzaeg P. Carcinoma of the nasopharynx. Histopathological examination with supplementary immunohistochemistry. Histopathology 1987;11:1161-9.

8 Wieland LH. The histological spectrum of nasopharyngeal carcinoma. In: de-The G, Ho Y, eds. Proceedings of carcinoma. In: de-The G, Ho Y, eds. Proceedings of international symposium on nasopharyngeal carcinoma: Etiology and Control. Lyon: Inte
Research on Cancer, 1978:41-50.

9 Liang PC, Chen CC, Chu CC, Hu YF, Chu HM, Tsung HM. The histologic classification, biological characteristics and histogenesis of nasopharyngeal carcinoma. Chinese Med f 1962;81:629-58.

$10 \mathrm{Li} \mathrm{CC}$, Pan QC, Chen CC. Hyperplasia and metaplasia in the nasopharynx. In: Li CC, Pan QC, Chen CC, eds. Nasopharyngeal carcinoma, clinical and laboratory researches. Gwangzhao: Gwangdong Scientific Technical Publications, 1983:143-53 (in Chinese).

11 Zong YS. Histopathology of nasopharyngeal carcinoma. In: Au PC, Zheng Y, eds. Aetiology and pathophysiology of nasopharyngeal carcinoma. Beijing: People's Health Pubnasopharyngeal carcinoma. Beijing:
lication, 1983:65-75 (in Chinese).

12 Zong YS, Li QX. Histopathology of paracancerous nasopharyngeal carcinoma in situ. Chinese Med $f$ 1986;99:763-71.

13 Lee JCK, Suen MWN. Intraepithelial neoplasia in mucosa of human nasopharyngeal carcinoma. In: Abstracts of the XII International Congress of the International Academy of Pathology and 7th World Congress of Academic and Environmental Pathology. Vienna: IAP, German Division, 1986:148.

14 Lee JCK. Precancerous changes. In: van Hasselt, CA, ed. Nasopharyngeal carcinoma. Hong Kong: Chinese University Press, 1991:37-45.

15 Krutchkoff DJ, Eisenberg E, Anderson C. Dysplasia of oral mucosa: A unified approach to proper evaluation. Mod Pathol 1991;4:113-19.

16 Sham JST, Wei WI, Zong YS, Choy D, Guo Y-Q, Yan L, Lin $\mathrm{ZX}, \mathrm{Ng} \mathrm{MH}$. Detection of subclinical nasopharyngeal carcinoma by fibreoptic endoscopy and multiple biopsy. Lancet 1990;335:371-4

17 Sham JST, Wei WI, Nicholls J, Chan CW, Choy D. Extent of nasopharyngeal carcinoma involvement inside the nasopharynx: lack of prognostic value or local control. Cancer 1992;69:854-9. 